\title{
Bouturage de Cola acuminata (P. Beauv.) Schott \& Endl.: Influence du substrat, de la longueur et de la surface foliaire sur l'enracinement de boutures à Kisangani, RD Congo
}

Par A Paluku1* ; A Okungo'; M Bwama².

(1) Institut Facultaire des Sciences Agronomiques de Yangambi, IFA, RD Congo ;

(2) Université Pédagogique Nationale, UPN, Kinshasa, RD Congo ;

*Auteur correspondant : panapasteur2000@gmail.com; Tél. (+243) 992140413

Original submitted in on 22 ${ }^{\text {nd }}$ August 2017. Published online at www.m.elewa.org on $31^{\text {st }}$ March 2018 https://dx.doi.org/10.4314/jab.v123i1.4

\section{RESUME}

Objectifs : Le colatier est arbre fruitier d'importance capital en RD Congo en général et à Kisangani en particulier. Les personnes impliquées dans sa filière (producteurs, commerçants) se procurent des revenus non négligeables. Les noix de kola, appréciées dans le milieu, sont pour la plupart tirées des arbres dans la forêt qui pour l'ensemble est menacée par les activités anthropiques. II serait donc souhaitable de préserver cette espèce dans le milieu par la domestication. Pour cette fin, cette étude vise la multiplication de $C$. acuminata par bouturage.

Méthodologie et résultats : Les facteurs type des substrats, surface foliaire, longueur de la tige des boutures et l'emploi d'auxine Acide Indole Butyrique (AIB) ont été expérimentés. Les substrats utilisés sont le sable de rivière, la sciure de bois, les balles de riz ainsi que leurs combinaisons deux à deux. Les surfaces foliaires 25 et $37,5 \mathrm{~cm}^{2}$ et les longueurs de tiges $3 ; 5$ et $7 \mathrm{~cm}$ ont été mis en comparaison. Deux essais ont été conduits, le premier testant les types de substrats dans un dispositif monofactoriel en blocs complets randomisés, les autres facteurs conduits, au second essai, dans un dispositif tri-factoriel, split plot. Le substrat sable a permis d'avoir plus de boutures enracinées $(81,11 \pm 11,67 \%)$. Les combinaisons longueurs de tiges de boutures $7 \mathrm{~cm}$ avec surface foliaire $37,5 \mathrm{~cm}^{2}$-auxine AIB et surface foliaire $25 \mathrm{~cm}^{2}$-sans auxine AIB sont meilleures, soit $70 \%$ des boutures enracinées.

Conclusion et application de résultats: L'espèce $C$. acuminata s'accommode bien à la technique de multiplication végétative par bouturage. On a atteint jusqu'à $81 \%$ de boutures qui se sont enracinées. Le facteur type de substrat influence considérablement le taux d'enracinement. Les autres facteurs n'ont pas influencé significativement ce paramètre. Les boutures s'étant enracinées, les travaux ultérieurs devraient se focaliser sur les suivis in situ de la croissance et du développement des boutures enracinées. Par cette technique, il est possible d'obtenir suffisamment des plantules, à mettre à la disposition des producteurs, en vue de la domestication de Cola acuminata.

Mots clés : Bouturage, enracinement, Cola acuminata, substrats, longueur des tiges, surface foliaire 
Paluku et al., J. Appl. Biosci. 2018 Bouturage de Cola acuminata (P. Beauv.) Schott \& Endl. : Influence du substrat, de la longueur et de la surface foliaire sur l'enracinement de boutures à Kisangani, RD Congo

Cutting of Cola acuminata (P. Beauv.) Schott \& Endl. : Influence of substrate, length and leaf area on the rooting of cuttings in Kisangani, DR Congo

\section{ABSTRACT}

Objectives: The Cola is fruit tree of capital importance in The Democratic Republic of Congo in general and in Kisangani in particular. The people involved in its sector (producers, traders) earn a significant income. The kola nuts, appreciated in the country, are mostly taken from the trees in the forest which are currently threatened by the anthropic activities. It would therefore be desirable to preserve this species in the environment by domestication. For this purpose, this study aims at the multiplication of $C$. acuminata by cuttings.

Methodology and results: Substrate type factors, leaf area, stem length of cuttings and the Idol Butyric Acid (IBA) auxin were experimented. The substrates used are river sand, sawdust, rice husks and their combinations in pairs. The leaf surfaces 25 and $37.5 \mathrm{~cm}^{2}$ and the stems lengths $3 ; 5$ and $7 \mathrm{~cm}$ were compared. Two tests were carried out, the first testing the substrates types in a monofactorial device in randomized complete block, the other factors conducted, in the second trial, in a tri-factorial device, split plot. The sand substrate allowed more of cuttings rooted $(81.11 \pm 11.67 \%)$. The combinations lengths of cuttings $7 \mathrm{~cm}$ with leaf area $37,5 \mathrm{~cm}^{2}-$ AIB auxin and leaf area $25 \mathrm{~cm}^{2}$-without AIB auxin are better, $70 \%$ of rooted cuttings.

Conclusion and application of results: The species $C$. acuminata adapts well to the technique of vegetative propagation by cuttings. Up to $81 \%$ of cuttings were rooted. The substrate type factor greatly affects the rooting rate. The other factors did not significantly influence this parameter. Since the cuttings were rooted, subsequent work should focus on in situ monitoring of the growth and development of rooted cuttings. By this technique, it is possible to obtain sufficient seedlings, to be made available to producers, for the domestication of Cola acuminata.

Key words: Cutting, rooting, Cola acuminata, substrates, stem length, leaf area

\section{INTRODUCTION}

La République Démocratique du Congo (RD Congo), un vaste pays, situé au cœur de l'Afrique, est couvert en grande partie par la forêt. Celle-ci couvre 155,5 millions d'hectares (dont 99 millions d'hectares de forêt dense humide), soit $67 \%$ du territoire national, $10 \%$ des forêts mondiales et près de $50 \%$ des forêts tropicales d'Afrique ; c'est la deuxième plus vaste forêt tropicale au monde (De Wasseige et al, 2009). Ce pays abrite cinq sites du patrimoine mondial de l'UNESCO et se place cinquième au rang mondial du point de vue de la diversité de la faune et de la flore, premier en diversité de mammifères et d'oiseaux (Kahindo, 2011). La forêt tropicale humide contient outre les produits forestiers ligneux, les produits forestiers non ligneux (PFNLs) d'une importance capitale pour les populations riveraines (Delbroux et al, 2007). Ces derniers englobent le gibier, les ressources halieutiques, les plantes médicinales, les fruits sauvages, les matériaux de construction et le bois de chauffe, qui sont les sources de produits utiles divers : tanins, résines, huiles, racines, feuilles, écorces, fruits, noix. Dans la province de la Tshopo où se trouve la ville de Kisangani, des études ont démontré que le Cola acuminata joue un rôle socio-économique important. Il est utilisé pour plusieurs fins sociales et procure un revenu non négligeable aux ménages concernés par sa commercialisation ou sa production. Son importance n'est pas à démontrer car il est visible dans tous les marchés de la ville (Evarest, 2008 ; Termote, 2012) et en vente ambulatoire dans les différents bistrots. La production de noix de cola se fait pour la plus grande partie par ramassage en forêt, alors que cette dernière subit de menaces de déforestation, qui du reste n'épargne pas la destruction de l'espèce $C$. acuminata. Si les dispositions ne sont pas prises pour la mis en culture de cette espèce, sa disparition est certaine. La domestication suppose l'adoption des techniques de multiplication adaptée à l'espèce (Leakey et Simons, 1998 ; Tchoundjeu et al., 2002a). La multiplication d'une espèce peut se faire soit générativement (multiplication par graine) soit végétativement (multiplication par autres parties de la plante que la 
graine), Beniest (1987). L'utilisation de graines pose les problèmes de la non reproductibilité des caractères de la plante mère à la plante fille (Tindall, 1967 ; Dupriez et De Leener, 1987), la perte du pouvoir germinative en cas de conservation des graines et le retard d'entrée en production des plants. Ainsi l'alternative serait entre outre la multiplication végétative (bouturage, marcottage, greffage et culture in vitro), Degrande et Facheux (2002). Pour ce qui concerne la kola, s'ajoute encore le problème de la concurrence entre l'utilisation de noix pour la multiplication et la consommation. Cette étude se propose d'évaluer l'une de méthodes de multiplication végétative à l'occurrence le bouturage pour la production de plants de $C$. acuminata à Kisangani. Des recherches antérieures sur plusieurs espèces d'arbres tropicaux ont indiquées une large gamme de facteurs influençant l'enracinement de boutures dans les conditions de propagateur. Ces facteurs incluent les génotypes, le type de substrat,

\section{MATERIELS ET METHODES}

Milieu d'étude : Les expérimentations ont été conduites au hangar du projet PAF/TSHOPO (projet des Produits Agro-forestiers pour l'Afrique) à l'Institut Facultaire des Sciences Agronomiques de Yangambi (IFA) situé au quartier Plateau Médical, commune Makiso $\left(0^{\circ} 30^{\prime} \mathrm{N}\right.$, $25^{\circ} 9^{\prime} \mathrm{E}, 390 \mathrm{~m}$ ) à Kisangani. La ville de Kisangani est caractérisée par un climat chaud et humide du type Africain (Af) selon la classification de Köppen. Les températures et les précipitations moyennes annuelles sont respectivement, de $25^{\circ} \mathrm{C}$ et $1800 \mathrm{~mm}$ (Nyongombe, 1993).

Matériels : L'espèce $C$. acuminata est un arbre de la famille de Sterculiaceae dont la tige peut atteindre $20 \mathrm{~m}$ de haut et $50 \mathrm{~cm}$ de diamètre. Les fruits sont de $300 \mathrm{~g}$ en moyenne et contiennent 1 - 12 graines (Lejoly et al., 2010 ; Vivien et Faure, 1996). Les boutures ont été collectionnées sur des arbres répertoriés dans la ville (à la Faculté des Sciences de l'Université de Kisangani : $0^{\circ} 30^{\prime} \mathrm{N}, 25^{\circ} 12^{\prime} \mathrm{E}, 398 \mathrm{~m}$ ) et aux alentours de la ville (à 25 $\mathrm{km}$ sur l'axe Lubutu: $\left.0^{\circ} 26^{\prime} \mathrm{N}, 25^{\circ} 22^{\prime} \mathrm{E}, 428 \mathrm{~m}\right)$. Les boutures récoltées sont humidifiées, mises dans un sachet puis introduites dans un sac enfin de faciliter le transport en moto jusqu'au lieu de bouturage.

Méthodes : Les expérimentations ont été conduites dans des propagateurs sans nébuliseurs installés au hangar $\mathrm{du}$ projet PAF/TSHOPO. Ces derniers sont des caisses simples en bois de $3 \mathrm{~m}$ de long, $1 \mathrm{~m}$ de large, 0,75 et $1 \mathrm{~m}$ la surface foliaire, la longueur de boutures, le type et la concentration d'auxines, le nombre et la position de nœuds pour les espèces Khaya ivorensis (Acajou d'Afrique) et Lovoa trichilioides (Dibétou, African Walnut), Tchoundjeu (1989); Prinus africana (Prunier d'Afrique, Pygeum, African plum tree), Tchoundjeu et al. (2002b), Avana (2006); Dacryodes edulis (Safoutier), Mialoundama et al. (2002); Irvingia gabonensis (Mangue sauvage, African mango, Mueba), Schiembo et al. (1997a); Ricinodendron heudelotii (Njansang, Corkwood, Erimado), Schiembo et al. (1997b) ; Pausinystallia johimbe(Yohimbé), Ngo Mpeck et al. (2003); Allanblackia floribunda (Ntia, Nyonne, Nsangomo), Atangana et al. (2006) et Nauclea diderrichii (Bilinga, Opepe, Ngulu-maza), Caspa et al. (2009). Dans la présente étude, les facteurs type des substrats, surface foliaire, longueur de la tige de boutures et l'emploi d'auxine AIB sont mis en examen.

de haut respectivement en avant et en arrière. La caisse, divisée en trois compartiments de $1 \mathrm{~m}^{2}$ chacun, est couverte par le papier plastique transparent de manière à rendre le système complètement étanche et imperméable. La base de propagateurs est constituée par une double couche de film de polyéthylène protégée par du sable fin. Ce dernier est recouvert successivement d'une couche de pierres suivie d'une couche de graviers de $10 \mathrm{~cm}$ d'épaisseur chacune. Le substrat d'enracinement repose sur la couche de gravier et son alimentation en eau se fait par capillarité. Le système ainsi décrit permet de créer un environnement humide en permanence (> $80 \%$ d'humidité) et une température de $28-30^{\circ} \mathrm{C}$ favorables au bon développement des boutures. Un tuyau en plastique d'environ $3 \mathrm{~cm}$ de diamètre et 30 $\mathrm{cm}$ de longueur inséré à l'angle de chaque compartiment, reposant sur la couche des pierres, permet d'ajuster quotidiennement le niveau d'eau dans le châssis (Tchoundjeu, 1989). Pour atteindre notre objectif, deux expérimentations ont été menées comparant :

(1) Le type de substrat : six traitements, sable ; sciure de bois ; balles de riz ; les combinaisons sable-balles de riz ; sable-sciure de bois et balles de riz-sciure de bois ;

(2) Combinaison $2 \times 2 \times 3$, auxines AIB (auxine et pas d'auxine), surfaces foliaires ( 25 et $37,5 \mathrm{~m}^{2}$ ) et longueur de la tige des boutures $(3 ; 5$ et $7 \mathrm{~cm})$. La combinaison de ces facteurs donne douze traitements. Pour l'essai sur le 
Paluku et al., J. Appl. Biosci. 2018 Bouturage de Cola acuminata (P. Beauv.) Schott \& Endl. : Influence du substrat, de la longueur et de la surface foliaire sur l'enracinement de boutures à Kisangani, RD Congo

type de substrat, le dispositif expérimental était les blocs complets randomisés avec 21 boutures par parcelle (soit un total de 21 boutures $\times 6$ traitements $\times 3$ répétitions $=$ 378 boutures). Pour l'essai sur la combinaison d'auxines AIB $x$ surfaces foliaires $x$ longueurs de la tige de boutures, le substrat utilisé est le sable fin avec un dispositif split plot, 10 boutures par parcelle (soit 10 boutures $\times 12$ traitements $\times 3$ répétitions $=360$ boutures). La stérilisation des substrats se faisait par grillage sur un demi-fût métallique chauffé au feu. Le substrat était chaque fois tourné en ajoutant régulièrement un peu d'eau pour permettre une bonne répartition de la chaleur dans la matière. Les boutures collectées sur des arbres différents étaient reparties équitablement sur les différents traitements.
L'hormone utilisée a été le RHIZOPON ${ }^{\circledR} A A$, une poudre contenant $2 \%$ d'acide indole butyrique (AIB). L'application se faisait par le contact de la base de la bouture avec la poudre. Durant l'expérimentation, les feuilles de boutures sont humidifiées par de très fines gouttes d'eau au moins une fois par jour à l'aide d'un pulvérisateur.

Collecte de données: Les observations se faisaient chaque semaine à partir de la deuxième semaine. Les paramètres prisent en compte sont le nombre de boutures morte, de boutures enracinées, le nombre et la longueur de racines formées. Le test statistique par l'ANOVA a permis de déterminer les différences entre les traitements. Le logiciel SPSS 14,0 a été utilisé pour les analyses statistiques. Les barres d'erreurs représentent l'erreur standard.
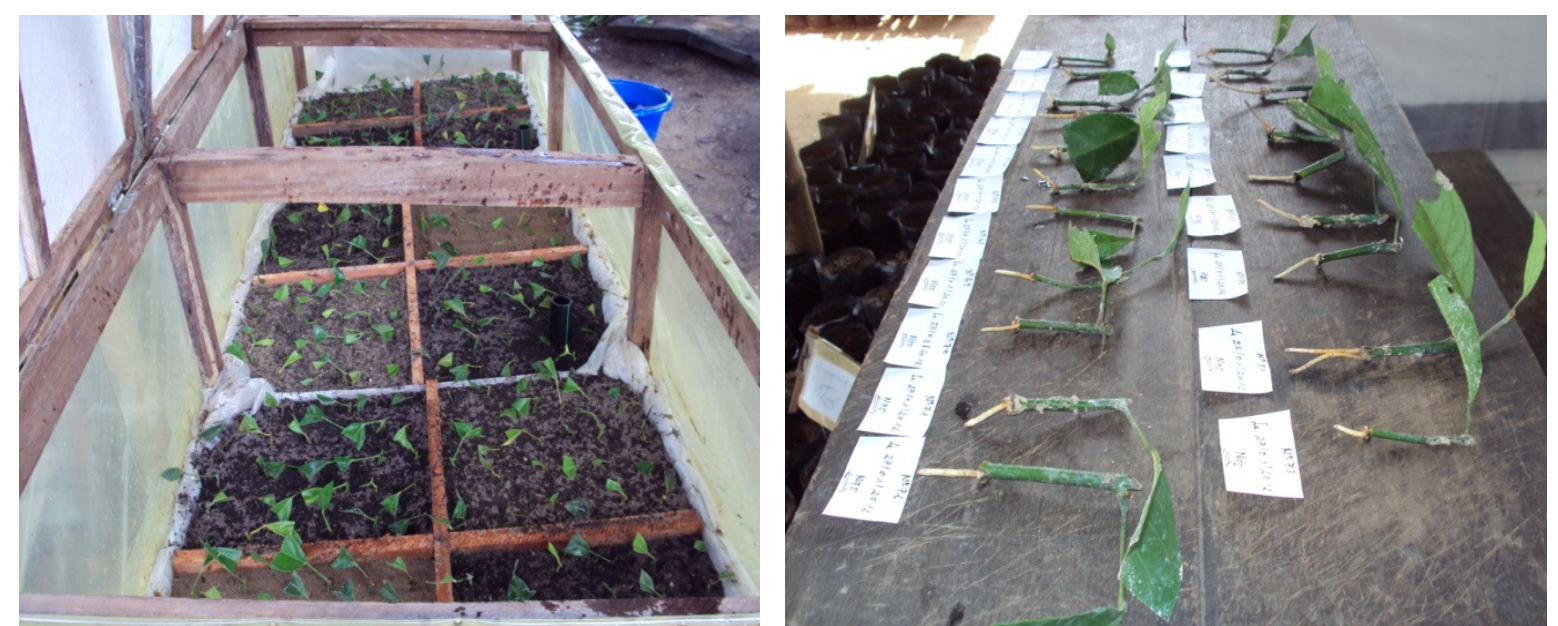

Figure 1. Boutures de $C$. acuminata dans le propagateur (gauche) et enracinées (droite)

\section{RESULTATS}

$1^{\circ}$ Influence du substrat sur l'enracinement de boutures: Le taux d'enracinement le plus élevé est obtenu avec le substrat sable, $81,11 \pm 11,67 \%$ (figure 2 ). Le substrat qui suit est la sciure de bois, 43,33 $\pm 21,79 \%$, sa combinaison avec le sable permet une amélioration de $\pm 67 \%$ (soit de 43,33 à $72,22 \%$ ). Les balles de riz n'ont pas donné des boutures enracinées. Ses combinaisons avec les autres substrats ont permis d'avoir des boutures enracinées (3,33 et $6,67 \%$ respectivement avec la sciure de bois et le sable). Le nombre de racines formées a varié de 1 à 2 (moyenne de 1,12 $\pm 0,32$ ) et la longueur de racines a varié de 0,5 à $5,0 \mathrm{~cm}$ avec moyenne de 1,73 \pm 1,10 (Figure 3). 


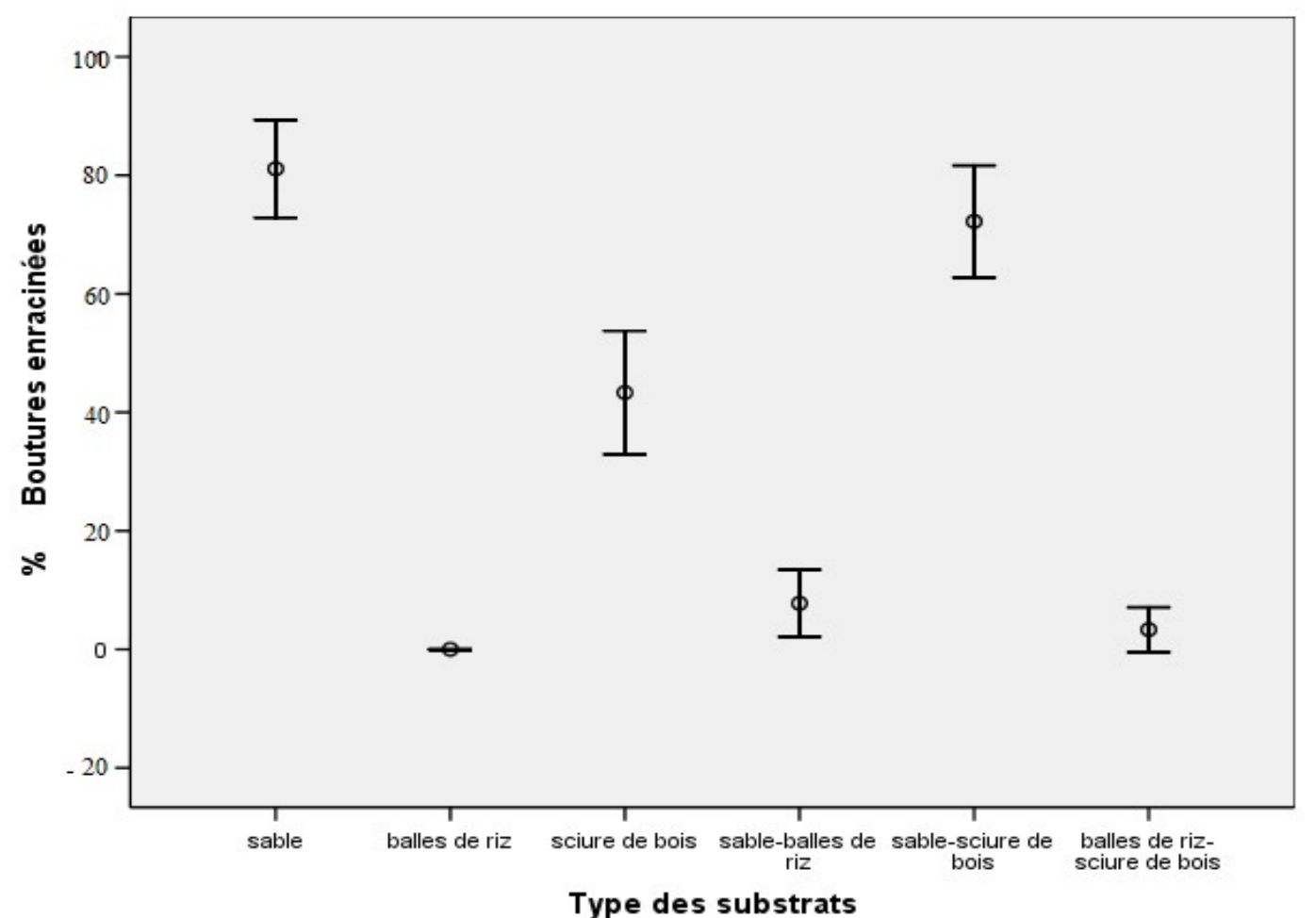

Figure 2. Taux d'enracinement des boutures en fonction de type des substrats

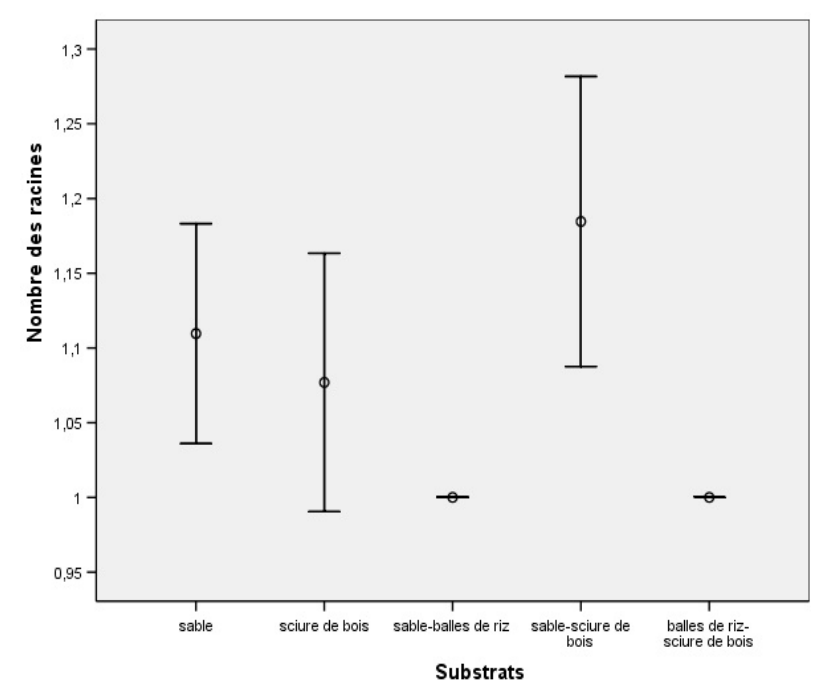

(a)

Figure 3. Nombre (a) et longueur (b) de racine des boutures de Cola acuminata

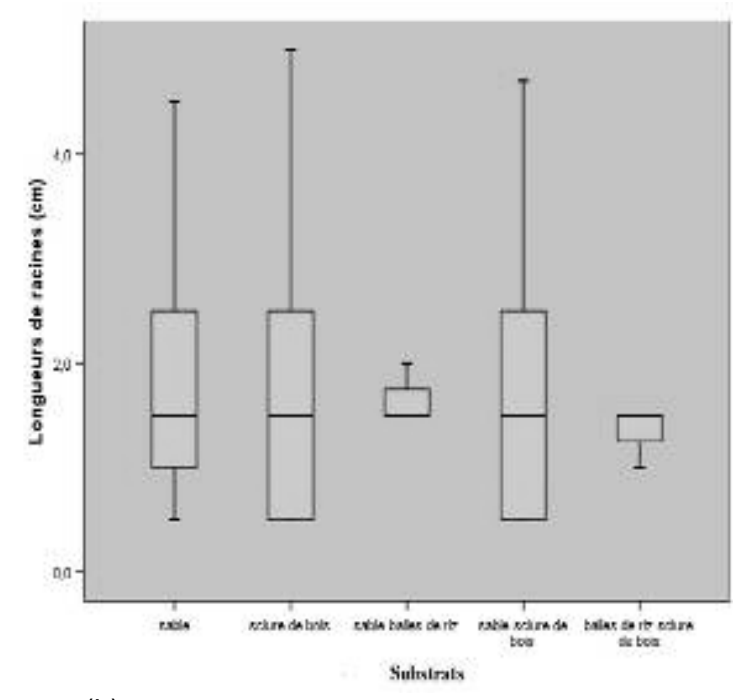

(b)
Le test statistique par l'analyse de variance (ANOVA) a montré de différences très hautement significatives de taux d'enracinement entre les différents substrats $(P<0,001)$ alors que le nombre de racines $(P=0,338)$ et la longueur de racines $(\mathrm{P}=0,868)$ ne différent pas significativement. $2^{\circ}$ Influence de la combinaison auxine AIB-surface foliaire-longueur de la tige de boutures sur l'enracinement de boutures $(\mathrm{H} 0=$ pas d'auxine $A I B ; H 1=$ auxine $A I B ; S 2=25 \mathrm{~cm}^{2}$; $S 3=$ $37,5 \mathrm{~cm}^{2} ; L 1=3 \mathrm{~cm} ; L 2=5 \mathrm{~cm}$ et $\left.L 3=7 \mathrm{~cm}\right)$. 


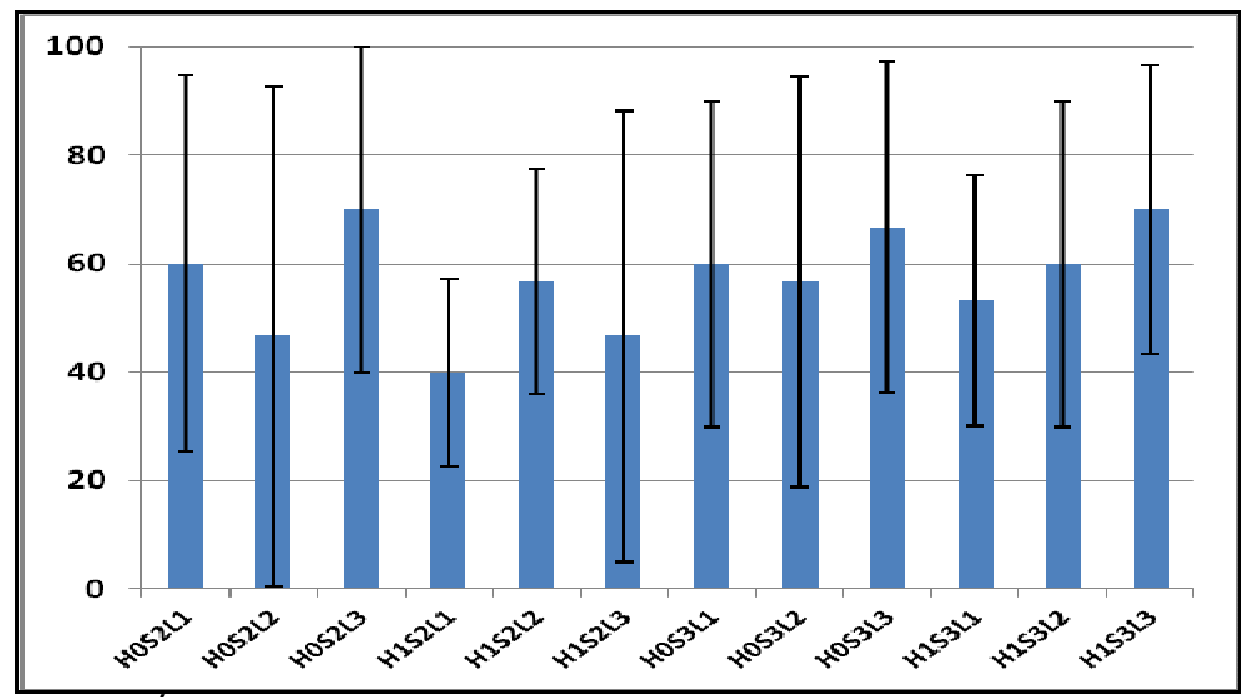

Figure 4. Évolution du taux d'enracinement des boutures de $C$. acuminata en fonction des combinaisons de facteurs

Les taux d'enracinement ont varié d'un traitement à l'autre. Les traitements avec les longueurs de la tige de boutures $7 \mathrm{~cm}$ combinés à l'auxine AlB-surface foliaire $37,5 \mathrm{~cm}^{2}$ et pas d'auxine AIB-surface foliaire $25 \mathrm{~cm}^{2}$ ont donné plus de boutures enracinées que les autres traitements, soit 70\% (Figure 4). Les taux d'enracinement évoluent dans les mêmes sens que les facteurs surfaces foliaires et longueurs de boutures. La surface foliaire $37,5 \mathrm{~cm}^{2}$ donne $61,17 \pm 6,30 \%$ des boutures enracinées, supérieur à $53,50 \pm 10,89 \%$ représentant le taux d'enracinement des boutures ayant $25 \mathrm{~cm}^{2}$. Les boutures de longueurs des tiges $3 ; 5$ et $7 \mathrm{~cm}$ donnent respectivement $53,25 \pm 9,42 ; \quad 55,25 \pm 5,67$ et $63,50 \pm 11,09 \%$ des taux d'enracinement (tableau 1).

Tableau 1. Taux d'enracinement (\%) des boutures de C. acuminata en fonction des surfaces foliaires et longueurs de la tige de boutures

\begin{tabular}{|c|c|c|c|c|c|c|}
\hline \multicolumn{7}{|c|}{ Longueurs de la tige de boutures } \\
\hline $\begin{array}{l}\text { Surfaces } \\
\text { Foliaires }\end{array}$ & Auxines AIB & $3 \mathrm{~cm}$ & $5 \mathrm{~cm}$ & $7 \mathrm{~cm}$ & Moyenne & $\begin{array}{l}\text { Coefficient de } \\
\text { Variation }\end{array}$ \\
\hline $25 \mathrm{~cm}^{2}$ & $\begin{array}{l}\text { Sans auxine } \\
\text { Avec auxine }\end{array}$ & $\begin{array}{l}60 \\
40\end{array}$ & $\begin{array}{l}47 \\
57\end{array}$ & $\begin{array}{l}70 \\
47\end{array}$ & $53,50 \pm 10,89$ & 20,36 \\
\hline $37,5 \mathrm{~cm}^{2}$ & $\begin{array}{l}\text { Sans auxine } \\
\text { Avec auxine }\end{array}$ & $\begin{array}{l}60 \\
53\end{array}$ & $\begin{array}{l}57 \\
60\end{array}$ & $\begin{array}{l}67 \\
70\end{array}$ & $61,17 \pm 6,30$ & 10,31 \\
\hline \multicolumn{2}{|c|}{$\begin{array}{l}\text { Moyenne } \\
\text { Coefficient de Variation }\end{array}$} & $\begin{array}{l}53,25 \pm 9,42 \\
17,71\end{array}$ & $\begin{array}{l}55,25 \pm 5,67 \\
10,28\end{array}$ & $\begin{array}{l}63,50 \pm 11,09 \\
17,47\end{array}$ & & \\
\hline
\end{tabular}

De tous les traitements effectués (facteurs seuls ou en combinaison), il n'a pas été observé des différences significatives $(P>0,005)$ tel que le montre le tableau 2. Aucun de facteurs, auxines AIB, surfaces foliaires, longueurs de la tige de boutures ainsi que leurs interactions bi- ou trifactorielles, n'a influencé d'une façon significative le taux d'enracinement des boutures. 
Paluku et al., J. Appl. Biosci. 2018 Bouturage de Cola acuminata (P. Beauv.) Schott \& Endl. : Influence du substrat, de la longueur et de la surface foliaire sur l'enracinement de boutures à Kisangani, RD Congo

Tableau 2. Analyse de la variance du taux d'enracinement des boutures de $C$. acuminata en fonction des différents facteurs et leurs combinaisons

\begin{tabular}{l|c|c|c|c|c}
\hline Sources de variation & SCE & df & CM & F & Sig.(5\%) \\
\hline Traitements & 2,922 & 11 &, 266 & 1,085 &, 372 \\
Auxines AIB &, 278 & 1 &, 278 & 1,135 &, 288 \\
Surfaces foliaires &, 544 & 1 &, 544 & 2,224 &, 137 \\
Longueurs de Boutures &, 689 & 2 &, 344 & 1,407 &, 246 \\
Auxines AIB * Surfaces foliaires &, 278 & 1 &, 278 & 1,135 &, 288 \\
Auxines AIB * Longueurs des Boutures &, 689 & 2 &, 344 & 1,407 &, 246 \\
Surfaces foliaires * Longueurs de Boutures &, 022 & 2 &, 011 &, 045 &, 956 \\
Auxines AIB * Surfaces foliaires * Longueurs de Boutures &, 422 & 2 &, 211 &, 862 &, 423 \\
Erreur & 85,200 & 348 &, 245 & & \\
\hline Total & 88,122 & 359 & & & \\
\hline
\end{tabular}

\section{DISCUSSION}

Les différents substrats mis en étude, montrent des différences sur la stimulation de l'enracinement chez l'espèce $C$. acuminata. De ces trois substrats, le sable a donné un taux d'enracinement très élevé comparativement aux autres substrats (sciure de bois et balles de riz). Des nombreuses études, comparant différents substrats, menées dans les conditions similaires (propagateur), ont montré que différentes espèces ont différentes préférence pour le sable (Vochysia hondurensis, Leakey et al., 1990 ; Cordia alliodora, Mesén et al., 1997a ; Garcinia kola, Nyansi, 2004 ; Allanblackia floribunda, Atangana et al., 2006 et Baillonella toxisperma, Ngo Mpeck et Atangana, 2007), la sciure de bois (Milicia excelsa, Ofori et al., 1996 ; Irvingia gabonensis, Shiembo et al., 1997a; Ricinodendron heudelotii, Shiembo et al., 1997b; Dacryodes edulis, Mialoundama et al., 2002 ; Prunus africana, Tchoundjeu et al., 2002b ; Pausinystalia johimbe, Tchoundjeu et al., 2004 et Diospyros classiflora, Tsobeng et al., 2011) ou le mélange d'entre eux (Eucalyptus deglupta, Leakey et al., 1990). Les différences de taux d'enracinement des boutures des espèces différentes en fonction des substrats ne pas bien élucidées (Leakey et al., 1990 ; Mesén et al., 1997b ; Tchoundjeu et al., 2002b), toute fois, les raisons seraient attribuées à la capacité de rétention en eau, à la circulation de l'air, au pH et la porosité du substrat. Ces facteurs affectent la respiration des tissus et la différentiation cellulaire à la base des boutures (Leakey et Newton, 1994). Une différence majeure entre ces substrats est leur capacité de rétention en eau (sable $=15 \%$, sable + sciure de bois $=36 \%$ ), et par conséquent leur rapport air / eau (sable $=2,1$; sable + sciure de bois $=0,5)$, Leakey et al., (1990). La porosité du substrat est nécessaire pour fournir suffisamment d'oxygène pour la respiration (Tchoundjeu et al., 2002b). Mesén et al. (1997b) a trouvé que pour Cordia alliodora, la conductance stomatique était plus élevée dans le sable que dans la sciure de bois, suggérant que la teneur en eau plus élevée de la sciure de bois inhibe la diffusion de l'oxygène et que l'asphyxie cause la mort des tissus et par le fait la réduction de l'absorption d'eau. On peut donc supposer que les espèces avec une préférence pour la sciure de bois sont celles mieux adaptées aux conditions saturées d'eau. Par conséquent, les espèces de tropiques humides peuvent être mieux adaptées à l'enracinement sous sciure de bois que les espèces de tropiques secs. La combinaison du sable avec les autres substrats permet d'améliorer leur qualité, la sciure de bois est passée de 43 à $72 \%$ et les balles de riz de 0 à $7 \%$. Ceci prouve à suffisance que le sable est le meilleur substrat d'enracinement pour l'espèce $C$. acuminata. Un substrat approprié pour l'enracinement de boutures doit en général avoir un volume optimal de pores et un taux de diffusion d'oxygène adéquat pour la respiration des racines des boutures (Andersen, 1986).Le nombre ainsi que la longueur de racines formées n'a pas varié significativement quelque soit les types de substrats, ces paramètres seraient des caractéristiques spécifiques.

Les taux d'enracinement évoluent dans les mêmes sens que les surfaces foliaires et les longueurs de la tige de boutures mais dans le sens contraire pour les applications d'auxines AIB. Toute fois il n'ya pas de variations importantes entre ces différents paramètres. Le taux d'enracinement de $C$. acuminata n'est pas influencé considérablement ni par la surface foliaire, ni par la longueur de boutures moins encore par l'application d'auxines. Les boutures de $C$. acuminata n'offrent pas beaucoup d'exigences pour son enracinement. 


\section{CONCLUSION}

Ces résultats montrent que le colatier, $C$. acuminata, offre une bonne appréciation à la multiplication végétative par bouturage. On a atteint même de taux supérieurs à $80 \%$ de boutures enracinées. Le substrat d'enracinement influence considérablement le taux d'enracinement. Le sable $(81,11 \%)$ s'est avéré le meilleur substrat à comparaison de sciure de bois $(43,33)$ et des balles de riz $(0,0 \%)$. La combinaison sable-sciure de bois a permis d'avoir un taux intermédiaire entre les deux substrats $(72,22 \%)$. Le taux d'enracinement est également fonction

\section{REMERCIEMENTS}

Nous remercions le « World Agroforestry Centre (West and Central Africa, Yaoundé, Cameroun )» qui à travers son projet "AFTP4A » a bien voulu soutenir financièrement les travaux de recherche. Nous

\section{RÉFÉRENCES BIBLIOGRAPHIQUES}

Andersen A.S., 1986. Stock plant conditions. In: Jackson M.B. (Ed.), New Root Formation in Plant and Cuttings. Martinus Njihoff, Dordrecht, pp. 223255.

Atangana AR, Tchoundjeu Z, Asaah EK, Simons AJ, Khasa DP, 2006. Domestication of Allanblackia floribunda: Amenability to vegetative propagation. Forest Ecology and Management 237: $246-251$.

Avana TM, 2006. Domestication de Prunus africana (Hook.f.) Kalkam (Rosaceae) : Étude de la germination et du bouturage. Thèse. Université de Yaoundé I, Yaoundé, Cameroun, 132p.

Beniest J, 1987. Guide pratique du maraîchage au Sénégal. CDH-Camberene, ISRA-FAO, éd. Saint-Paul, Dakar, Sénégal, 144p.

Caspa RG, Kuoudiekong L, Nwegueh BA, Djeudeng TS, Lahjou JC, Onana J, 2009. Effect of different substrates on rooting and shoot development of juvenile stem cuttings of Nuclea diderichiii (De Wild \& T. Durand) Merill. International Journal for Biological and Chemical Sciences 3 (5): 1124 $-1132$.

De Wasseige C, Devers D, De Marcken P, Eba'a Atyi R, Nasi R, Mayaux P, 2009. Les Forêts du Bassin du Congo - État des Forêts 2008. Office des publications de I'Union Européenne, Luxembourg, 426p. [online at http://www.observatoirecomifac.net/edf2008.php ].

Debroux L, Hart T, Kaimowitz D, Karsenty A, Topa G., 2007. Forest in post-conflict Democratic de la surface foliaire et de la longueur des boutures. La multiplication végétative pourrait être l'option favorable pour la propagation de $C$. acuminata, les graines (noix) étant en concurrence entre la consommation et la propagation. Les boutures enracinées doivent évoluer jusqu'à l'obtention d'arbres en production. Les recherches futures pourront être orientées vers le suivi in situ de la croissance et du développement des boutures enracinées de C. acuminata.

remercions également le Prof. Patrick Van Damme, de I'Université de Gant en Belgique pour sa contribution à l'élaboration de ce document par ses observations et remarques constructives.

Republic of Congo: Analysis of a Priority Agenda. CIFOR, the World Bank and CIRAD.

Degrande A. et Facheux C., 2002. Production et Commercialisation des plantes produites dans les pépinières villageoises. Guide pratique pour formateurs, encadreurs et pépiniéristes. ICRAFWest Africa. World Agroforestry Centre. 52p.

Dupriez $H$. et De Leener $P, 1987$. Jardins et vergers d'Afrique. Terre et Vie, Nivelles, Belgique, 354p.

Everaert G, 2008. Recherche Ethnobotanique : Étude de marché sur les Plantes Alimentaires Sauvages (R.D. Congo, Province Orientale). Résumé de Thèse. Université de Gent, Gent, Belgique, $31 \mathrm{p}$.

Kahindo 2011. Potentiel en Produits Forestiers Autres que le Bois d'oeuvre dans les formations forestières de la région de Kisangani. Cas des rotins Eremospatha haullevilleana De Wild. et Laccosperma secundiflorum (P. Beauv.) Küntze de la Réserve Forestière de Yoko (Province Orientale, RD Congo). Thèse de docteur en science, UNIKIS, 269p.

Leakey R.R.B. et Simons AJ, 1998. The domestication and commercialization of indigenous trees in Agrofrestry for the alleviation of poverty. Agroforestry System 38: 165-176.

Leakey RRB, Mésen JF, Tchoundjeu Z, Longman KA, Dick JMcP, Newton A, Martin A, Grace J, Munro RC. and Muthoka PN, 1990. Low technology techniques for the vegetative propagation of tropical trees. Commonwealth Forestry Review 69 (3): $247-257$. 
Paluku et al., J. Appl. Biosci. 2018 Bouturage de Cola acuminata (P. Beauv.) Schott \& Endl. : Influence du substrat, de la longueur et de la surface foliaire sur l'enracinement de boutures à Kisangani, RD Congo

Leakey RRB. and Newton AC, 1994. Domestication of "Cinderella" species as the start of a "woody plant revolution". In Leakey RRB and Newton AC (Eds); Tropical Trees: The Potential for Domestication and the Rebuilding of Forest Resources. HMSO, London, p. 3-6.

Lejoly J., Ndjele MB, Geerink D, 2010. Catalogue - Flore des plantes vasculaires des districts de Kisangani et de la Tshopo (RD Congo), $4^{e}$ éd. Revue et augmentée, incluant les clés et la distribution pour 70 familles, publiées dans la taxonomania, $30: 1-308$, Bruxelles.

Mesén JF, Newton AC, Leakey RRB, 1997. Vegetative propagation of Cordia alliodora (Ruiz \& Pavon) Oken: the effects of IBA concentration, propagation medium and cutting origin. Forest Ecology and Management 92: 45-54.

Mesén JF, Newton AC, Leakey RRB, 1997. The effects of propagation environment and foliar area on the rooting physiology of Cordia alliodora (Ruiz \& Pavon) Oken cuttings. Trees 11: 404-411.

Mialoundama F, Avana ML, Youmbi E, Mampouya PC, Tchoundjeu Z, Mbeuyo M, Galamo GR, Bell JM, Kopguep F, Tsobeng AC, Abega J, 2002. Vegetative propagation of Dacryodes edulis (G. Don) H. Lam by marcotts, cutting and micropropagation. Journal of Forests, Trees and livelihoods 12 (1): 85-96.

Ngo-Mpeck ML, Tchoundjeu Z, Asaah E, 2003. Vegetative propagation of Pausinystalia johimbe (K. Schum) by leafy stem cutting. Propagation of Ornamental Plants 3 (2): 11 - 18.

Ngo-Mpeck ML. and Atangana A, 2007. Rooting of Leafy Stem Cuttings of Baillonella toxisperma. Forest Science 53 (5): 571-579.

Nyansi HAD, 2004. Multiplication végétative de Garcinia kola Heckel: effet du substrat de propagation et de la surface foliaire sur la rhizogenèse des boutures de tige. Mémoire de fin d'études d'Ingénieur des Eaux, Forêts et Chasse. FASA, Université de Dschang, Dschang, Cameroun, 42 p.

Nyongombe U, 1993. Contribution à l'étude écologique et biologique des poissons de la rivière MASINDULA (affluent de la Tshopo) à Kisangani. Thèse de doctorat, IFA-Yangambi, 193p.

Ofori DA, Newton AC, Leakey RRB, Grace J, 1996. Vegetative propagation of Milicia excelsa by leafy stem cuttings: effects of auxin concentration, leaf areas and rooting medium. Forest Ecology and Management 84: 39-48.

Shiembo PN, Newton AC, Leakey RRB, 1997 . Vegetative propagation of Irvingia gabonensis, a West African fruit tree. Forest Ecology and Management 87: 185-192.

Schiembo PN, Newton AC, Leakey RRB, 1997b. Vegetative propagation of Ricinodendron heudelotii, a West African fruit tree. Journal of Tropical Forest Science 9 (4): $514-525$.

Tchoundjeu Z, Kengeu J, Leakey RRB, 2002a. Domestication of Dacryodes edulis: State-of-theArt. Forests, trees and livelihoods, 12: 3-13

Tchoundjeu Z, Avana ML, Leakey RRB, Simons AJ, Asaah E, Duguma B, Bell JM, 2002b. Vegetative propagation of Prunus africana: Effect of rooting medium, auxin concentrations and leaf area. Agroforestry Systems 54: 183- 192.

Tchoundjeu Z, Ngo Mpeck ML, Asaah E, Amougou A, 2004. The role of vegetative propagation in the domestication of Pausinystalia johimbe (K. Schum), a highly threatened medicinal species of West and Central Africa. Forest Ecology and management.

Tchoundjeu Z, 1989. Vegetative Propagation of the Tropical Hardwoods of Khaya ivorensis (A. Chef) and Lovoa trichilioides (Harm). Thesis Submitted to the University of Edinburgh for the Degree of Doctor of Philosophy, 261p.

Termote C, 2012. Wild edible plant use in Tshopo District, Democratic Republic of Congo. PhD thesis. Faculty of Bioscience Engineering, University of Ghent, Belgium, 294p.

Tindall H.D, 1968. Fruits et légumes en Afrique Occidentale. FAO, Rome, Italie, 260p.

Tsobeng A, Tchoundjeu Z, Kouodiekong L, Asaah E, 2011. Effective propagation of Diospyros crassiflora (Hiern) using twing cuttings. International Journal of Biosciences 1 (4) : 109117.

Vivien J. and Faure JJ, 1996. Fruitiers Sauvages d'Afrique (Espèces du Cameroun). Ed. NguilaKerou, les presses de l'imprimerie Téqui Saintcénéré, $416 \mathrm{p}$. 